\title{
Locating Battery Charging Stations to Facilitate Almost Shortest Paths*
}

\author{
Esther M. Arkin ${ }^{1}$, Paz Carmi ${ }^{2}$, Matthew J. Katz ${ }^{2}$, Joseph S. B. \\ Mitchell $^{1}$, and Michael Segal ${ }^{3}$
}

1 Department of Applied Mathematics and Statistics

Stony Brook University, USA

\{estie, jsbm\}@ams.stonybrook.edu

2 Department of Computer Science

Ben-Gurion University, Israel

\{carmip,matya\}@cs.bgu.ac.il

3 Department of Communication Systems Engineering, Ben-Gurion University, Israel

segal@bgu.ac.il

\begin{abstract}
We study a facility location problem motivated by requirements pertaining to the distribution of charging stations for electric vehicles: Place a minimum number of battery charging stations at a subset of nodes of a network, so that battery-powered electric vehicles will be able to move between destinations using " $t$-spanning" routes, of lengths within a factor $t>1$ of the length of a shortest path, while having sufficient charging stations along the way. We give constantfactor approximation algorithms for minimizing the number of charging stations, subject to the $t$-spanning constraint. We study two versions of the problem, one in which the stations are required to support a single ride (to a single destination), and one in which the stations are to support multiple rides through a sequence of destinations, where the destinations are revealed one at a time.
\end{abstract}

1998 ACM Subject Classification G.2.2 Graph Theory: Network Problems; F.2.2 Nonnumerical Algorithms and Problems: Geometrical problems and computations

Keywords and phrases approximation algorithms; geometric spanners; transportation networks

Digital Object Identifier 10.4230/OASIcs.ATMOS.2014.25

\section{Introduction}

Network optimization problems ask us to construct "good" networks subject to various constraints and objectives. There is always a trade-off between the cost of the network and its functionality. For example, in the problem of computing an optimal spanning subgraph, there is a trade-off between the objectives of having a low cost network in terms of the number or weight of the edges and the preservation of shortest path distance in the subgraph, when compared to shortest path distance in the full graph. Specifically, given an edge-weighted

\footnotetext{
* E. Arkin, P. Carmi, M. Katz and J. Mitchell are partially supported by grant 2010074 from the United States - Israel Binational Science Foundation. E. Arkin and J. Mitchell are partially supported by NSF (CCF-1018388). P. Carmi is partially supported by grant 680/11 from the Israel Science Foundation. M. Katz is partially supported by grant $1045 / 10$ from the Israel Science Foundation. M. Segal is partially supported by General Motors Inc., by the Israeli Ministry of Defense and by an EPSRC grant from the United Kingdom Research Council.
}

cc) (i) Esther M. Arkin, Paz Carmi, Matthew J. Katz, Joseph S. B. Mitchell, and Michael Segal; 14th Workshop on Algorithmic Approaches for Transportation Modelling, Optimization, and Systems (ATMOS'14). Editors: Stefan Funke and Matúš Mihalák; pp. 25-33

OpenAccess Series in Informatics

OASICS Schloss Dagstuhl - Leibniz-Zentrum für Informatik, Dagstuhl Publishing, Germany 
graph $G=(V, E)$ and a real number $t \geq 1$, a $t$-spanner of $G$ is a spanning subgraph $G^{\prime}$ with the property that for each edge $\{x, y\}$ in $G$, there exists a path between $x$ and $y$ in $G^{\prime}$ whose weight is no more than $t$ times the weight of the edge $\{x, y\}$. Such a path is said to be a $t$-spanning path.

Constructing good spanners arises in transportation network design, since it is important for networks of roads or rails to provide efficient routes between pairs of locations, thereby minimizing the total travel time for cars and trains while minimizing environmental impact in terms of energy consumption and air pollution. Environmental awareness has prompted the demand for "green energy" approaches to transportation, industrial production, and daily life. A key component of such approaches to the transportation sector is the use of electric and hybrid automobiles and trucks, in place of vehicles that require the combustion of fossil fuels.

In this paper we address spanner optimization problems that are motivated by the need for infrastructure in support of electric vehicles. Instead of measuring cost in terms of the number or weight of edges, we consider a cost in terms of the number of nodes selected. Specifically, we consider the problem of placing a minimum number of battery charging stations at a subset of nodes of a network, so that battery-powered electric vehicles will be able to move between destinations using routes that are provably close to being shortest paths, while having sufficient charging stations along the way.

We use a simple model of motion in which we assume distances between pairs of points is measured by (or approximated by) Euclidean $\left(L_{2}\right)$ distance. However, our methods can be applied to other metric spaces, such as the $L_{1}$ distance (measuring "Manhattan" driving distances in a regular grid of streets) or more general road networks.

\subsection{Related work}

A variant of our (basic) problem has been studied by Storandt and Funke [11]. On the one hand, they studied the problem in a more general setting, where the underlying network is modeled by a weighted directed graph, but, on the other hand, they require only the existence of a path between any pair of destinations (in either one or both directions), while we require the existence of a light (i.e., short) path between any pair of destinations.

The battery charging station location problem (when requiring only the existence of a path) is closely related to the problem of computing a connected dominating set. Let $L>0$ be the distance that the vehicle can travel without recharging its battery when starting with a full battery. Given a set $P$ of points in the plane, let $G$ be the graph over $P$ in which there is an edge between $p, q \in P$ if and only if $|p q| \leq L$, and assume that $G$ is connected. Then a minimum Connected Dominated Set (CDS) of $G$ corresponds to a minimum set of battery charging stations in $G$, and vice versa.

The problem of finding a minimum CDS in a unit disk graph has been shown to be NP-complete [3]. Marathe et al. [8] present a 10-approximation centralized algorithm for this problem. Cheng et al. [2] present a polynomial-time approximation scheme that guarantees an approximation factor of $(1+1 / s)$ with running time of $n^{O\left((s \log s)^{2}\right)}$. In addition, the distributed construction of a small CDS has attracted significant attention. The first such algorithm due to Wan et al. [13] has an approximation factor of 8 and running time $O(n)$. However, the analysis of [13] ignores delays incurred by interference (in the context of wireless networks). An algorithm given in [10] computes, with high probability, an $O(1)$-approximation in $O\left(n \log ^{2} n\right)$ time and explicitly handles interference. This algorithm is based on a distance-2-coloring (D2-coloring), where no two nodes at 2-hop distance can have the same color. Funke et al. [4] improved the approximation ratio to 6.91. Subsequently, 
the approximation ratio was further improved in $[5,7,14,15]$, and it currently stands at 6 [12].

In short, without the additional requirement that there exist a $t$-spanning path between any two destinations, the problem is a well studied problem with good approximation results.

There are numerous papers dealing with geometric spanners; see the book by Narasimhan and Smid [9] for an extensive survey. However, the constructions that are most relevant to the construction described in this paper are those that use cones, e.g., the Yao-graph [16], the $\theta$-graph [6], and the stable roommates spanner [1]. The main idea in these constructions is to partition the plane around each input point into $k$ equal-angle cones, and to pick a "closest" point in each of these cones, where the definition of "closest" varies in each construction.

\subsection{Definitions and results}

Let $P$ be a set of $n$ points (locations) in the plane, and let $L$ be the distance that the vehicle can travel without recharging its battery when starting with a full battery. Without loss of generality we assume that $L=1$. For $r>0$, denote by $U D G_{r}(P)$ the graph whose vertices are the points of $P$ and there exists an edge between two vertices if and only if the Euclidean distance between their corresponding points is at most $r$. Let $G=U D G_{1}(P)$, i.e., $G$ is the Unit Disk Graph induced by $P$. We assume that $G$ is connected, since, otherwise, there is no solution to our problem.

For a subset $Q \subseteq P$, we denote by $G_{Q}$ the graph $G$, such that the battery charging stations are located at the vertices corresponding to the points of $Q$. Let $\pi_{G_{Q}}(p, q)=\prec p=$ $p_{1}, \ldots, p_{k}=q \succ$ be a path between $p$ and $q$ in $G_{Q}$. We say that $\pi_{G_{Q}}(p, q)$ is legal if and only if the following two conditions are satisfied: (i) $p_{i} \in Q, 1<i<k$, and (ii) $\left|p_{i} p_{i+1}\right| \leq 1$, $1 \leq i \leq k-1$. Let $\delta_{G_{Q}}(p, q)$ represent a legal shortest path between $p$ and $q$ in $G_{Q}$; its length is denoted by $\left|\delta_{G_{Q}}(p, q)\right|$. If such a path does not exist, then $\left|\delta_{G_{Q}}(p, q)\right|=\infty$. We are ready to state the two main problems that are studied in this paper, where BCS stands for Battery Charging Station. We believe that the second one models reality quite well.

BCS Location Problem (single ride). Given a set $P$ of points in the plane and a constant $t>1$. Locate as few battery charging stations as possible at points of $P$, such that, for any two points $p, q \in P,\left|\delta_{G_{Q}}(p, q)\right| \leq t \cdot\left|\delta_{G_{P}}(p, q)\right|$, where $Q$ is the subset of points of $P$ at which battery charging stations have been located. In other words, find a minimum cardinality subset $Q \subseteq P$, such that, if one places battery charging stations at the points of $Q$, then, for any two points $p, q \in P$, the distance that a vehicle at $p$ (with a fully charged battery) would have to travel in order to reach $q$ is not much longer than the distance it would travel if there were a battery charging station at each point of $P$. We are assuming that whenever the vehicle passes through a battery charging station its battery is recharged.

BCS Location Problem (multiple rides). Given a set $P$ of points in the plane and a constant $t>1$. Find a minimum cardinality subset $Q \subseteq P$, such that, if one places battery charging stations at the points of $Q$, then the following requirement is satisfied. Let $p$ be any starting point and let $\sigma=\left(p=q_{0}, q_{1}, \ldots, q_{l}\right)$ be a sequence of destinations in $P$, where destination $q_{i}, 1 \leq i \leq l$, is revealed only once destination $q_{i-1}$ has been reached. Then, for any $1 \leq i \leq l$, given the next destination $q_{i}$, one can compute a path $\hat{\pi}_{G_{Q}}\left(q_{i-1}, q_{i}\right)$ (in $G_{Q}$ ) from $q_{i-1}$ to $q_{i}$, such that $\Sigma_{j=1}^{i}\left|\hat{\pi}_{G_{Q}}\left(q_{j-1}, q_{j}\right)\right| \leq t \cdot \Sigma_{j=1}^{i} \delta_{G_{P}}\left(q_{j-1}, q_{j}\right)$. Or, in words, the total distance traveled so far, where the destinations are given one by one and the battery is recharged only at points of $Q$ is not much longer than the distance traveled so far, where there is a battery charging station at each point of $P$. We are assuming that at the beginning (when the vehicle is at the starting point $p$ ), the vehicle's battery is 


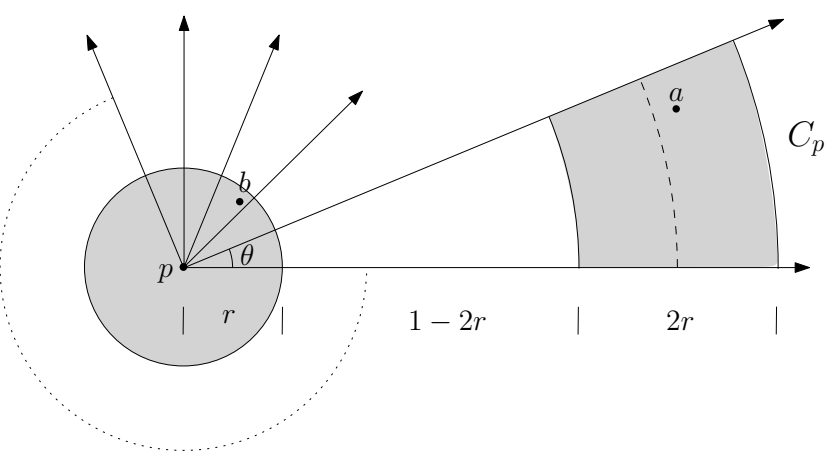

Figure 1 Locating battery charging stations for cone $C_{p}$, where $p \in M I S$. The regions $C_{p} \cap$ $\left(D_{1+r}(p) \backslash D_{1-r}(p)\right)$ and $D_{r}(p)$ are in gray, and $|a b| \leq 1$.

fully charged, but afterwards it is recharged only when the vehicle passes through a battery charging station.

Let $m$ be the size of an optimal solution to the weaker version of the single ride problem, where one needs to locate as few charging stations as possible, so that for any two points in $P$ there is a legal path between them (but not necessarily a legal $t$-spanning path). We show how to compute a subset $Q \subseteq P$, which will be used for both our problems. We then prove that $|Q|=O(\mathrm{~m})$, immediately implying that $Q$ is a constant-factor approximation of the optimal solutions for these problems. After computing $Q$, we describe how given a pair of points, in the single ride version, or the next destination, in the multiple rides version, to compute a legal path in $G_{Q}$ such that the appropriate length requirement is satisfied. Finally, we show that $Q$ can be computed in $O(n \log n)$ time.

\section{Single ride}

Let $r=r(t)<1$ be some constant dependent on $t$ that will be specified later. We begin by finding a maximal independent set, $M I S$, in $U D G_{r}(P)$ and locating battery charging stations at the vertices of $M I S$. This does not yet guarantee that there exists a $t$-spanning path between any two points as required, so we need to locate additional stations. We shall denote the disk of radius $\rho$ centered at point $p$ by $D_{\rho}(p)$. Let $\theta$ be an angle such that $\frac{2 \pi}{\theta}$ is an integer and $\sin \frac{\theta}{2} \leq \frac{r}{2}$. Set $k=\frac{2 \pi}{\theta}$. For each point $p \in M I S$, partition the plane into $k$ cones with apex $p$ and angle $\theta$. Now, for each of $p$ 's cones, $C_{p}$, if there exist a pair of points $a \in P \cap C_{p} \cap\left(D_{1+r}(p) \backslash D_{1-r}(p)\right)$ and $b \in P \cap D_{r}(p)$ such that $|a b| \leq 1$, then locate battery charging stations at $a$ and $b$ for one such pair; see Figure 1.

The following observation follows from the requirement $\sin \frac{\theta}{2} \leq \frac{r}{2}$.

- Observation 1. For any two points $x$ and $y$ in $C_{p} \cap\left(D_{1+r}(p) \backslash D_{1-r}(p)\right)$, it holds that $|x y| \leq 3 r$.

Let $Q$ be the set of points where battery charging stations have been located. We prove that (in $G_{Q}$ ) there exists a $t$-spanning path between any two points.

- Theorem 2. For any $p, q \in P,\left|\delta_{G_{Q}}(p, q)\right| \leq t \cdot \mid \delta_{G_{P}}(p, q)$.

Proof. If $|p q| \leq 1$, then $\left|\delta_{G_{Q}}(p, q)\right|=|p q|=\left|\delta_{G_{P}}(p, q)\right|$. Thus, we assume that $|p q|>1$. Let $\delta_{G_{P}}(p, q)$ be $\prec p=p_{1}, p_{2}, \ldots, p_{k-1}, p_{k}=q \succ$, where $k>2$ (since $|p q|>1$ ). For each $p \in P$, let $p^{\prime}$ denote a point in $Q$ (possibly $p$ itself) such that $\left|p p^{\prime}\right| \leq r$. Such a point always exists since otherwise we can add $p$ to $M I S \subseteq Q$. 
Observation 3. (i) $\left|p_{i}^{\prime} p_{i+1}\right| \leq 1+r$, and (ii) if $\left|p_{i}^{\prime} p_{i+1}^{\prime}\right|>1$, then $\left|p_{i}^{\prime} p_{i+1}\right|>1-r$.

Proof. By the triangle inequality, we obtain the upper bound $\left|p_{i}^{\prime} p_{i+1}\right| \leq\left|p_{i}^{\prime} p_{i}\right|+\left|p_{i} p_{i+1}\right| \leq$ $1+r$.

By the triangle inequality, we have $\left|p_{i}^{\prime} p_{i+1}^{\prime}\right| \leq\left|p_{i}^{\prime} p_{i+1}\right|+\left|p_{i+1} p_{i+1}^{\prime}\right| \leq\left|p_{i}^{\prime} p_{i+1}\right|+r$, or $\left|p_{i}^{\prime} p_{i+1}\right| \geq\left|p_{i}^{\prime} p_{i+1}^{\prime}\right|-r$. And since $\left|p_{i}^{\prime} p_{i+1}^{\prime}\right|>1$, we obtain the lower bound.

Now, we build a legal path $\pi_{G_{Q}}(p, q)$ from $p$ to $q$ in $G_{Q}$. The path $\pi_{G_{Q}}(p, q)$ starts at $p=p_{1}$, ends at $q=p_{k}$, and visits points $p_{1}^{\prime}, p_{2}^{\prime}, \ldots, p_{k}^{\prime}$.

For each $i, 1 \leq i \leq k-1$, we distinguish between two cases, according to the distance $\left|p_{i}^{\prime} p_{i+1}^{\prime}\right|$ :

Case (1): $\left|\mathbf{p}_{\mathbf{i}}^{\prime} \mathbf{p}_{\mathbf{i}+1}^{\prime}\right| \leq \mathbf{1}$. Then, the path $\pi_{G_{Q}}(p, q)$ visits point $p_{i+1}^{\prime}$ immediately after point $p_{i}^{\prime}$. The length of this direct (legal) path from $p_{i}^{\prime}$ to $p_{i+1}^{\prime}$ is

$$
\left|p_{i}^{\prime} p_{i+1}^{\prime}\right| \leq\left|p_{i}^{\prime} p_{i}\right|+\left|p_{i} p_{i+1}\right|+\left|p_{i+1} p_{i+1}^{\prime}\right| \leq\left|p_{i} p_{i+1}\right|+2 r .
$$

Case (2): $\left|\mathbf{p}_{\mathbf{i}}^{\prime} \mathbf{p}_{\mathbf{i}+1}^{\prime}\right|>\mathbf{1}$. Let $C_{p_{i}^{\prime}}$ be the cone with apex $p_{i}^{\prime}$ that contains $p_{i+1}$ and proceed as follows. By Observation 3, we have $p_{i+1} \in\left(C_{p_{i}^{\prime}} \cap\left(D_{1+r}\left(p_{i}^{\prime}\right) \backslash D_{1-r}\left(p_{i}^{\prime}\right)\right)\right)$. Moreover, $p_{i} \in D_{r}\left(p_{i}^{\prime}\right)$ and $\left|p_{i} p_{i+1}\right| \leq 1$. Thus, $Q$ includes battery charging stations at points $a$ and $b$ such that $a \in C_{p_{i}^{\prime}} \cap\left(D_{1+r}\left(p_{i}^{\prime}\right) \backslash D_{1-r}\left(p_{i}^{\prime}\right)\right)$ and $b \in D_{r}\left(p_{i}^{\prime}\right)$. (Possibly $a=p_{i+1}$, and possibly $b=p_{i}^{\prime}$.) Therefore, our constructed legal path from $p_{i}^{\prime}$ to $p_{i+1}^{\prime}$ is defined to be $\prec p_{i}^{\prime}, b, a, p_{i+1}^{\prime} \succ$.

The length of our constructed path $\prec p_{i}^{\prime}, b, a, p_{i+1}^{\prime} \succ$ is

$$
\left|p_{i}^{\prime} b\right|+|b a|+\left|a p_{i+1}^{\prime}\right| \leq\left|p_{i}^{\prime} b\right|+|b a|+\left|a p_{i+1}\right|+\left|p_{i+1} p_{i+1}^{\prime}\right| \leq r+1+3 r+r=1+5 r,
$$

where the inequality $\left|a p_{i+1}\right| \leq 3 r$ follows from Observation 1 . Since we are assuming that $\left|p_{i}^{\prime} p_{i+1}^{\prime}\right|>1$, and we know that $\left|p_{i}^{\prime} p_{i+1}^{\prime}\right| \leq\left|p_{i} p_{i+1}\right|+2 r$, we get that

$$
\left|p_{i} p_{i+1}\right| \geq\left|p_{i}^{\prime} p_{i+1}^{\prime}\right|-2 r>1-2 r,
$$

implying that the length of our constructed path $\prec p_{i}^{\prime}, b, a, p_{i+1}^{\prime} \succ$ is

$$
\left|p_{i}^{\prime} b\right|+|b a|+\left|a p_{i+1}^{\prime}\right| \leq 1+5 r \leq\left|p_{i} p_{i+1}\right|+7 r .
$$

Thus, in both cases, the length of our constructed legal path from $p_{i}^{\prime}$ to $p_{i+1}^{\prime}$ is at most $\left|p_{i} p_{i+1}\right|+7 r$. Thus, the length, $\left|\pi_{G_{Q}}\left(p_{i}^{\prime}, p_{i+2}^{\prime}\right)\right|$, of our constructed path from $p_{i}^{\prime}$ to $p_{i+2}^{\prime}$ is, for any $1 \leq i \leq k-2$, at most $\left|p_{i} p_{i+1}\right|+\left|p_{i+1} p_{i+2}\right|+14 r$, implying the following bound on the dilation:

$$
\frac{\left|\pi_{G_{Q}}\left(p_{i}^{\prime}, p_{i+2}^{\prime}\right)\right|}{\left|p_{i} p_{i+1}\right|+\left|p_{i+1} p_{i+2}\right|} \leq \frac{\left|p_{i} p_{i+1}\right|+\left|p_{i+1} p_{i+2}\right|+14 r}{\left|p_{i} p_{i+1}\right|+\left|p_{i+1} p_{i+2}\right|} \leq 1+\frac{14 r}{\left|p_{i} p_{i+2}\right|}<1+14 r .
$$

The last inequality above comes from the optimality of the path $\delta_{G_{P}}(p, q)$, which implies that for each $i, 1 \leq i \leq k-2,\left|p_{i} p_{i+2}\right|>1$ (otherwise the path would go directly from $p_{i}$ to $\left.p_{i+2}\right)$.

Overall, then, considering the partition of $\delta_{G_{P}}(p, q)$ into the subpaths $\delta_{G_{P}}\left(p_{i}, p_{i+2}\right)$, for $i=1,3,5, \ldots$, and comparing to the lengths $\left|\pi_{G_{Q}}\left(p_{i}^{\prime}, p_{i+2}^{\prime}\right)\right|$ of each of the associated constructed subpaths, we get an overall dilation factor of at most $1+14 r$ (assuming $k$ is odd). Finally, taking into account the case where $k$ is even and the initial and final steps from $p_{1}$ to $p_{1}^{\prime}$ and from $p_{k}^{\prime}$ to $p_{k}$, we get a bound of $1+18 r$ on the dilation factor. Thus, we pick $r=(t-1) / 18$. 
Let $O P T$ be an optimal solution to the weaker version of the problem, where one needs to locate as few charging stations as possible, so that for any two points in $P$ there is a legal path between them (but not necessarily a legal $t$-spanning path). Moreover, denote by $M I S\left(U D G_{r}(P)\right)$ and $M C D S\left(U D G_{r}(P)\right)$ a maximal independent set and a minimum connected dominating set of $U D G_{r}(P)$, respectively.

Theorem 4. The number of battery charging stations in our solution is bounded by a constant times $|O P T|$, i.e., $|Q|=O(|O P T|)$.

Proof. Observe first that $O P T$ is a connected dominating set of $U D G_{1}(P)$ and therefore $|O P T| \geq\left|M C D S\left(U D G_{1}(P)\right)\right|$. It is well known that $\left|M I S\left(U D G_{1}(P)\right)\right|=O\left(\left|M C D S\left(U D G_{1}(P)\right)\right|\right)$; actually, $\left|M I S\left(U D G_{1}(P)\right)\right| \leq 3.8\left|M C D S\left(U D G_{1}(P)\right)\right|+1.2$, see [15]. We conclude that $\left|M I S\left(U D G_{1}(P)\right)\right|=O(|O P T|)$. On the other hand, the number of battery charging stations that we locate is $|Q|=O\left(\frac{1}{t-1}\left|M I S\left(U D G_{r}(P)\right)\right|\right)=O\left(\frac{1}{r^{2}(t-1)}\left|M I S\left(U D G_{1}(P)\right)\right|\right)=$ $O\left(\frac{1}{(t-1)^{3}}|O P T|\right)$.

\section{Multiple rides}

In this section we extend the basic version of the problem considered in the previous section to a more general and more realistic setting. In the general version, we are given a sequence of points (i.e., destinations), rather than a single destination, and we need to visit them one after the other. However, the points are given to us one at time; that is, the next destination is given to us only when the current destination has been reached. Moreover, in contrast with the basic version, we cannot assume that the vehicle's battery is fully charged at the beginning of the $i$ th trip (except for the first trip); rather, its battery level depends on the distance traveled from the last charging station visited. Under these more general and natural conditions, we would like to achieve similar goals.

More precisely, let $P$ be a set of points in the plane and let $t>1$ be a constant. We wish to find a minimum cardinality subset $Q \subseteq P$, such that, if one places battery charging stations at the points of $Q$, then the following requirement is satisfied. Let $p$ be any starting point and let $\sigma=\left(p=q_{0}, q_{1}, \ldots, q_{l}\right)$ be a sequence of destinations in $P$, where destination $q_{i}$, $1 \leq i \leq l$, is revealed only once destination $q_{i-1}$ has been reached. Then, for any $1 \leq i \leq l$, given the next destination $q_{i}$, one can compute a path $\hat{\pi}_{G_{Q}}\left(q_{i-1}, q_{i}\right)$ (in $G_{Q}$ ) from $q_{i-1}$ to $q_{i}$, such that $\sum_{j=1}^{i}\left|\hat{\pi}_{G_{Q}}\left(q_{j-1}, q_{j}\right)\right| \leq t \cdot \sum_{j=1}^{i} \delta_{G_{P}}\left(q_{j-1}, q_{j}\right)$. Or, in words, the total distance traveled so far, where the destinations are given one by one and the battery is recharged only at points of $Q$ is not much longer than the distance traveled so far, where there is a battery charging station at each point of $P$. We are assuming that at the beginning (when the vehicle is at the starting point $p$ ), the vehicle's battery is fully charged, but afterwards it is recharged only when the vehicle passes through a battery charging station.

We prove below that the set $Q$, computed in the previous section, is also suitable for the general version. That is, by placing charging stations at the points of $Q$, we are able to satisfy the requirement concerning the distance traveled so far. Notice that, by using the same set $Q$ as in the previous section, the requirement concerning the size of $Q$ is already satisfied (i.e., $|Q|$ is bounded by some constant times $|O P T|$ ), since the size of an optimal solution to the multiple ride version is clearly at least the size of an optimal solution to the single ride version.

- Theorem 5. For any $1 \leq i \leq l$, given the next destination $q_{i}$, one can compute a path $\hat{\pi}_{G_{Q}}\left(q_{i-1}, q_{i}\right)$ (in $G_{Q}$ ) from $q_{i-1}$ to $q_{i}$, such that $\Sigma_{j=1}^{i}\left|\hat{\pi}_{G_{Q}}\left(q_{j-1}, q_{j}\right)\right| \leq t \cdot \sum_{j=1}^{i} \delta_{G_{P}}\left(q_{j-1}, q_{j}\right)$. 
Proof. Given the next destination $q_{i}$, the general idea is to use the path computed in the previous section, unless this means that the car reaches $q_{i}$ with battery level less than $r$. More precisely, if $\left|q_{i-1} q_{i}\right| \leq 1-2 r$, then depending on whether we can reach $q_{i}$ with battery level at least $r$ or not, we drive directly from $q_{i-1}$ to $q_{i}$, or drive from $q_{i-1}$ to $q_{i}$ via $q_{i-1}^{\prime}$, where $q_{i-1}^{\prime}$ denotes a point in $Q$ such that $\left|q_{i-1} q_{i-1}^{\prime}\right| \leq r$. Notice that assuming the battery level at the beginning of the journey is at least $r$, the vehicle will complete the journey and reach $q_{i}$ with battery level at least $r$.

Now, consider the case where $\left|q_{i-1} q_{i}\right|>1-2 r$, and let $\delta_{G_{P}}\left(q_{i-1}, q_{i}\right)$ be $\prec q_{i-1}=$ $p_{1}, p_{2}, \ldots, p_{k-1}, p_{k}=q_{i} \succ$, where $k \geq 2$. (Notice that here, unlike in the previous section, it is possible that $k=2$.) In this case we drive along the path $\pi_{G_{Q}}\left(p_{1}, p_{k}\right)$, defined in the previous section. This path starts at $p_{1}$, ends at $p_{k}$, and visits points $p_{1}^{\prime}, \ldots, p_{k}^{\prime}$. Again, assuming the battery level at the beginning of the journey is at least $r$ and recalling that $\left|p_{1} p_{1}^{\prime}\right| \leq r$, the car will complete the journey, i.e., reach $p_{k}$. Moreover, since $\left|p_{k}^{\prime} p_{k}\right| \leq r$, the battery level at the end of the journey is at least $1-r \geq r$.

We denote the constructed path from $q_{i-1}$ to $q_{i}$ by $\hat{\pi}_{G_{Q}}\left(q_{i-1}, q_{i}\right)$. It remains to prove that for a sufficiently small constant $r, \Sigma_{j=1}^{i}\left|\hat{\pi}_{G_{Q}}\left(q_{j-1}, q_{j}\right)\right| \leq t \cdot \Sigma_{j=1}^{i} \delta_{G_{P}}\left(q_{j-1}, q_{j}\right)$. We say that path $\hat{\pi}_{G_{Q}}\left(q_{j-1}, q_{j}\right)$ is short, for $1 \leq j \leq i$, if $\left|q_{j-1} q_{j}\right| \leq 1-2 r$; otherwise it is long. By the analysis of the previous section, we know that for any $1 \leq j \leq i$, if $\hat{\pi}_{G_{Q}}\left(q_{j-1}, q_{j}\right)$ is long, then its dilation factor is bounded by $1+18 r$.

We partition the sequence of paths $\hat{\pi}_{G_{Q}}\left(q_{0}, q_{1}\right), \ldots, \hat{\pi}_{G_{Q}}\left(q_{i-1}, q_{i}\right)$ into maximal subsequences, such that in each subsequence either all paths are short or all paths are long. Consider a subsequence $\Pi$ of the former kind and let $q_{\min }$ be the starting point of the first path in $\Pi$ and let $q_{\max }$ be the ending point of the last path in $\Pi$. Since all the paths in $\Pi$ are short, we know that the length of the shortest path in $G_{P}$ starting at $q_{\text {min }}$, passing through $q_{\min +1}, \ldots, q_{\max -1}$, and ending at $q_{\max }$ is simply $x=\sum_{j=\min }^{\max -1}\left|q_{j} q_{j+1}\right|$. Therefore the number of detours (to a charging station) that $\Pi$ makes is at most $\left\lceil\frac{x}{1-2 r}\right\rceil$. Notice that if $\Pi$ is the whole sequence $\hat{\pi}_{G_{Q}}\left(q_{0}, q_{1}\right), \ldots, \hat{\pi}_{G_{Q}}\left(q_{i-1}, q_{i}\right)$, then, if $x \leq 1-r$, then the dilation factor of $\Pi$ is 1 , and otherwise, it is less than

$$
\frac{x+2 r\left\lfloor\frac{x}{1-2 r}\right\rfloor}{x} \leq 1+\frac{2 r}{1-2 r}=\frac{1}{1-2 r} .
$$

If $\Pi$ is not the whole sequence, then each subsequence of short paths is followed and/or preceded by a subsequence of long paths. We thus charge the first detour in each subsequence of short paths to one of its adjacent subsequences. This increases the bound on the dilation factor of a subsequence of long paths to $1+22 r$, and allows us to bound the dilation factor of a subsequence of short paths by $\frac{1}{1-2 r}$. Since $r \leq 1 / 3$, the dilation factor of the whole sequence is bounded by $1+22 r$, and by fixing $r \leq \frac{t-1}{22}$, the requirement concerning the distance traveled so far is satisfied.

\section{Running time}

In this section we show how to compute in $O(n \log n)$ time the set $Q$ of points at which we locate battery charging stations. Recall that we first compute a maximal independent set, $M I S$, in $U D G_{r}(P)$. This can be done in $O(n \log n)$ time. (E.g., select a point $p \in P$ that has not been considered yet, and compute the distance between $p$ and each of the $O(1)$ points of MIS lying in the axis-parallel square of edge length $2 r$ around $p$. If for each of these points the distance is greater than $r$, then add $p$ to $M I S$. Move to the next unconsidered point in $P$.) Now, for each $p \in M I S$, we need to consider the set $A_{p} \subseteq P$ of points in the annulus centered at $p$ with radii $1-r$ and $1+r$, and partition it into $k$ subsets, corresponding to the 
$k$ cones with apex $p$. For each such subset $A_{p}^{i}$, we need to find a pair $a \in A_{p}^{i}$ and $b \in D_{r}(p)$, such that $|a b| \leq 1$ (if such a pair exists). However, we cannot afford to preprocess $P$ for annulus reporting queries. Instead, we proceed as follows. We preprocess $P$ for axis-parallel square reporting queries. Given a point $p \in M I S$, we perform a query with the axis-parallel square of edge length $2(1+r)$ centered at $p$. Let $S_{p}$ be the query's output. Now, for each point $q \in S_{p}$, we check whether its distance from $p$ is within the range $[1-r, 1+r]$, and, if yes, we add it to the subset $A_{p}^{i}$ to which it belongs. Similarly, we perform a reporting query with the square of edge length $2 r$ centered at $p$. For each point in the query's output, we check whether it lies in $D_{r}(p)$ or not. We now have the sets $P \cap D_{r}(p)$ and $A_{p}^{1}, \ldots, A_{p}^{k}$. Next, we construct the Voronoi diagram of the set $P \cap D_{r}(p)$ and preprocess it for efficient point location queries. It remains to find the pairs $\{a, b\}$, for each of the sets $A_{p}^{1}, \ldots, A_{p}^{k}$. Consider the set $A_{p}^{1}$. For each point $q \in A_{p}^{1}$, we find its nearest neighbor in $P \cap D_{r}(p)$, until we encounter a pair whose corresponding distance is at most 1 (or we have finished checking all points in $A_{p}^{1}$ ). We now move to the next set $A_{p}^{2}$, etc.

We prove that the total running time of the algorithm described above for computing $Q$ is only $O(n \log n)$, assuming of course that $r$ is a constant. Notice first that if we apply the algorithm to a single point $p \in M I S$, then the running time is clearly $O\left(\left|S_{p}\right| \log \left|S_{p}\right|\right)$. We now prove that each point $q \in P$ belongs to at most some constant number (dependent on $r$ ) of the sets $S_{p}$, where $p \in M I S$.

- Claim 6. Let $q \in P$. Then $q$ belongs to at most $O\left(1 / r^{2}\right)$ sets $S_{p}$, where $p \in M I S$.

Proof. Notice that if we draw a disk of radius $r / 2$ around each of the points in $M I S$, then the resulting disks are pairwise disjoint. Now, $q \in S_{p}$, for $p \in M I S$, if and only if $p$ is in the square of edge length $2(1+r)$ centered at $q$. However, the observation above implies that the number of points of $M I S$ in the square around $q$ is only $O\left(1 / r^{2}\right)$.

The following theorem summarizes the main result of this section.

- Theorem 7. The set $Q$ can be computed in $O\left(\left(1 / r^{2}\right) n \log n\right)$ time.

- Remark. A more complex algorithm gives a better bound in which $1 / r^{2}$ is replaced by $1 / r$. However, since $r$ is a constant, we preferred to describe the simpler algorithm.

\section{References}

1 Prosenjit Bose, Paz Carmi, Lilach Chaitman-Yerushalmi, Sébastien Collette, Matthew J. Katz, and Stefan Langerman. Stable roommates spanner. Computational Geometry, 46(2):120-130, 2013.

2 Xiuzhen Cheng, Xiao Huang, Deying Li, Weili Wu, and Ding-Zhu Du. A polynomialtime approximation scheme for the minimum-connected dominating set in ad hoc wireless networks. Networks, 42(4):202-208, 2003.

3 Brent N. Clark, Charles J. Colbourn, and David S. Johnson. Unit disk graphs. Discrete Mathematics, 86(1-3):165-177, 1990.

4 Stefan Funke, Alexander Kesselman, Ulrich Meyer, and Michael Segal. A simple improved distributed algorithm for minimum cds in unit disk graphs. ACM Trans. Sen. Netw., 2(3):444-453, 2006.

5 Xiaofeng Gao, Yuexuan Wang, Xianyue Li, and Weili Wu. Analysis on theoretical bounds for approximating dominating set problems. Discrete Mathematics, Algorithms and Applications, 1(1):71-84, 2009.

6 J. Mark Keil and Carl A. Gutwin. Classes of graphs which approximate the complete euclidean graph. Discrete $\& 3$ Computational Geometry, 7(1):13-28, 1992. 
7 Minming Li, Peng-Jun Wan, and Frances Yao. Tighter approximation bounds for minimum CDS in wireless ad hoc networks. In International Symposium on Algorithms and Computation (ISAAC), pages 699-709. 2009.

8 M. V. Marathe, H. Breu, H. B. Hunt, S. S. Ravi, and D. J. Rosenkrantz. Simple heuristics for unit disk graphs. Networks, 25(2):59-68, 1995.

9 Giri Narasimhan and Michiel Smid. Geometric spanner networks. Cambridge University Press, 2007.

10 Srinivasan Parthasarathy and Rajiv Gandhi. Distributed algorithms for coloring and domination in wireless ad hoc networks. In IARCS Annual Conference on Foundations of Software Technology and Theoretical Computer Science (FSTTCS), pages 447-459. 2005.

11 Sabine Storandt and Stefan Funke. Enabling e-mobility: Facility location for battery loading stations. In 27th Conference on Artificial Intelligence (AAAI), 2013.

12 Alireza Vahdatpour, Foad Dabiri, Maryam Moazeni, and Majid Sarrafzadeh. Theoretical bound and practical analysis of connected dominating set in ad hoc and sensor networks. In 22nd International Symposium on Distributed Computing (DISC), pages 481-495, 2008.

13 Peng-Jun Wan, K.M. Alzoubi, and O. Frieder. Distributed construction of connected dominating set in wireless ad hoc networks. In 21st IEEE International Conference on Computer Communications (INFOCOM), volume 3, pages 1597-1604, 2002.

14 Peng-Jun Wan, Lixin Wang, and F. Yao. Two-phased approximation algorithms for minimum CDS in wireless ad hoc networks. In 28th International Conference on Distributed Computing Systems (ICDCS), pages 337-344, 2008.

15 Weili Wu, Hongwei Du, Xiaohua Jia, Yingshu Li, and Scott C.-H. Huang. Minimum connected dominating sets and maximal independent sets in unit disk graphs. Theoretical Computer Science, 352(1-3):1-7, 2006.

16 Andrew Chi-Chih Yao. On constructing minimum spanning trees in $k$-dimensional spaces and related problems. SIAM Journal on Computing, 11(4):721-736, 1982. 